\title{
Effects of Pulvinar Inactivation on Spatial Decision-making between Equal and Asymmetric Reward Options
}

\author{
Melanie Wilke ${ }^{1,2,3}$, Igor Kagan ${ }^{1,3}$, and Richard A. Andersen ${ }^{1}$
}

\begin{abstract}
The ability to selectively process visual inputs and to decide between multiple movement options in an adaptive manner is critical for survival. Such decisions are known to be influenced by factors such as reward expectation and visual saliency. The dorsal pulvinar connects to a multitude of cortical areas that are involved in visuospatial memory and integrate information about upcoming eye movements with expected reward values. However, it is unclear whether the dorsal pulvinar is critically involved in spatial memory and reward-based oculomotor decision behavior. To examine this, we reversibly inactivated the dorsal portion of the pulvinar while monkeys performed a delayed memory saccade task that included choices between
\end{abstract}

\section{INTRODUCTION}

Survival in an environment that contains a large amount of information requires organisms to focus on a subset of incoming sensory information and to strategically decide between multiple response options. Spatial working memory and the ability to direct eye movements to explore spatial locations of potential interest constitute important cognitive components of this selection process. Selection of eye movement targets is guided by both the visual saliency of the stimuli as well as motivational values (Opris \& Bruce, 2005). For example, primates typically saccade to stimuli that predict high reward and saccade parameters such as time of initiation, accuracy, and frequency of choices are influenced by the predicted reward outcome (Milstein \& Dorris, 2007; McCoy \& Platt, 2005; Lauwereyns, Watanabe, Coe, \& Hikosaka, 2002; Takikawa, Kawagoe, Itoh, Nakahara, \& Hikosaka, 2002).

Although much of the research on visuospatial processing and reward-based saccadic decision-making has focused on cortical areas, there is ample evidence that the dorsal pulvinar may play a critical role in these functions (Grieve, Acuna, \& Cudeiro, 2000). (1) Anatomical evidence: Unlike the ventral pulvinar, which is primarily interconnected with visual areas and receives input from the superficial layers of the superior colliculus (SC), the dorsal pulvinar has reciprocal connections with cortical

\footnotetext{
${ }^{1}$ California Institute of Technology, ${ }^{2}$ University of Goettingen, ${ }^{3}$ German Primate Center, Leibniz Institute for Primate Research, Goettingen, Germany
}

equally or unequally rewarded options. Pulvinar inactivation resulted in a delay of saccade initiation toward memorized contralesional targets but did not affect spatial memory. Furthermore, pulvinar inactivation caused a pronounced choice bias toward the ipsilesional hemifield when the reward value in the two hemifields was equal. However, this choice bias could be alleviated by placing a high reward target into the contralesional hemifield. The bias was less affected by the manipulation of relative visual saliency between the two competing targets. These results suggest that the dorsal pulvinar is involved in determining the behavioral desirability of movement goals while being less critical for spatial memory and reward processing.

areas that maintain spatial information for upcoming eye movements and are modulated by expected reward values (Kaas \& Lyon, 2007; Stepniewska, 2004). Specifically, the medial portion of the dorsal pulvinar, which we focus on in the current study, is reciprocally interconnected with the posterior parietal cortex, the upper bank of the STS (Cappe, Morel, \& Rouiller, 2007; Gutierrez, Cola, Seltzer, \& Cusick, 2000; Yeterian \& Pandya, 1989; Asanuma, Andersen, \& Cowan, 1985), the pFC (Gutierrez et al., 2000; Selemon \& Goldman-Rakic, 1988), and limbic structures such as the posterior parahippocampal gyrus and the posterior cingulate cortex (Baleydier \& Mauguiere, 1985). This connectivity with cortical areas known to be involved in the processing of behavioral relevance has led to the hypothesis that the pulvinar enhances the processing of biologically relevant stimuli by interaction with those regions (Padmala, Lim, \& Pessoa, 2010). (2) Physiological evidence: Firing rates and response variability of pulvinar neurons in monkeys are modulated by visual attention and perception (Wilke, Mueller, \& Leopold, 2009; Bender \& Youakim, 2001; Benevento \& Port, 1995; Robinson \& Petersen, 1992; Petersen, Robinson, \& Keys, 1985), and neuroimaging studies in humans demonstrated attention-related BOLD signals in the pulvinar (Hulme, Whiteley, \& Shipp, 2010; Padmala et al., 2010; Kastner et al., 2004). Pulvinar neurons exhibit presaccadic and postsaccadic response changes (Berman \& Wurtz, 2011; Benevento \& Port, 1995; Robinson, McClurkin, Kertzman, \& Petersen, 1991; Robinson, McClurkin, \& Kertzman, 1990), and visual responses are enhanced when a stimulus represents the goal of a saccade (Petersen 
et al., 1985). In addition, fMRI studies in humans and monkeys demonstrated BOLD modulation in the pulvinar during saccade execution (Krebs et al., 2010; Baker, Patel, Corbetta, \& Snyder, 2006). (3) Lesion evidence: Perhaps, the most convincing evidence for a critical role of the pulvinar in spatial orienting and eye movement decisions can be derived from lesion studies. Pulvinar lesions in humans (Arend, Rafal, \& Ward, 2008; Karnath, Himmelbach, \& Rorden, 2002; Danziger, Ward, Owen, \& Rafal, 2001; Rafal \& Posner, 1987; Zihl \& von Cramon, 1979) and monkeys (Wilke, Turchi, Smith, \& Leopold, 2010; Petersen, Robinson, \& Morris, 1987) lead to spatial neglect symptoms, including difficulties to direct attention toward the contralesional field. Although the causal role of the pulvinar in the selection of eye movement targets remains less explored, recent studies reported lesion-induced deficits in oculomotor integration and saccadic decision-making (Van der Stigchel, Arend, van Koningsbruggen, \& Rafal, 2010; Wilke, Turchi, et al., 2010; Arend, Machado, et al., 2008). Specifically, a previous study in monkeys that employed a visually guided saccade task showed that reversible inactivation of the dorsal pulvinar is associated with a choice bias toward the ipsilesional hemifield although performance toward single targets remained largely intact (Wilke, Turchi, et al., 2010).

The primary goal of the current study was to investigate how inactivation of the dorsal pulvinar influences spatial memory and reward-based oculomotor decision behavior. We were particularly interested to see whether the spatial choice bias following pulvinar inactivation that has been observed in the previous study (Wilke, Turchi, et al., 2010) occurs at the level of perceptual extinction or at later stages of the decision-making process. To this end, we employed a delayed memory saccade task with interleaved instructed (single target) and choice (bilateral targets) trials and varied the target-associated reward magnitude. In a subset of experiments, we also varied the visual saliency by using targets with different luminance levels. We hypothesized that, if the choice bias was primarily because of perceptual extinction, it would persist even when the stimulus in the contralesional field indicated a higher reward in comparison with the ipsilesional field. In contrast, if the biased spatial choice behavior was because of a motivational/intentional movement bias, we expected the high reward stimulus to increase the willingness to perform an eye movement toward the contralesional space.

\section{METHODS}

All surgical and animal care procedures were done in accordance with National Institutes of Health guidelines and were approved by the California Institute of Technology, Animal Care and Use Committee.

\section{Preparation}

Two male rhesus macaques (Macaca mulatta) weighing 8-10 kg were implanted with an MR-compatible plastic
(PEEK) headpost embedded in Palacos bone cement (BioMet) attached to the cranium with short ceramic screws (Thomas Recording), under general anesthesia. Monkeys were chronically implanted with a 22-gauge guide PEEK cannula (Plastics One, Roanoke, VA) penetrating the dura and targeting the dorsal pulvinar in the left hemisphere. The top portion of this guide cannula was set in a corrugated ceramic cylinder, which was affixed to the skull with ceramic screws and bone cement and served as a guide for (acute) insertion of the 28-gauge internal PEEK cannula during the experiments. Before each surgery, we acquired high-resolution anatomical MRI (see Structural MRI Acquisition). Anatomical MRI scans were transformed into an AC-PC plane, and cannula positions were planned by comparing the coronal MRI slices with the combined MRI and histology atlas of the macaque brain (Saleem \& Logothetis, 2006).

\section{Pulvinar Inactivation}

Microinfusions of the GABA-A agonist 4,5,6,7-tetrahydroisoxazolo[5,4-c]-pyridin-3-ol (THIP; Tocris Bioscience, Ellisville, MO) were made in each inactivation session via a sterile 28-gauge internal cannula. To confirm the injection locations, the MR contrast agent gadolinium (Magnevist, Berlex Imaging, Montville, NJ) was added to the solution of weak phosphate buffered saline, and anatomical MR images were acquired. The THIP was dissolved in PBS (along with the gadolinium), and the solution ( $\mathrm{pH}$ 7.0-7.5) was sterile filtered (Corning, Inc., Corning, NY) before injection. Specificity of drug effects was further validated in an additional control session in which only the vehicle and gadolinium were injected. Total injection volumes ranged from 2.0 to $4.5 \mu \mathrm{l}$ of $6.6 \mathrm{mg} / \mathrm{ml}$ of THIP, which were delivered at a rate of $0.7-1.0 \mu \mathrm{l} / \mathrm{min}$ using a $100-\mu \mathrm{l}$ gas-tight Hamilton syringe driven by a digital infusion pump (Harvard Apparatus, Holliston, MA). The infusions were carried out while the animals were awake and sitting in a primate chair, with their heads restrained via implanted head posts, before the MRI scanning. All injections in both monkeys were made in the left pulvinar. We conducted 13 inactivation sessions into the dorsal pulvinar in monkey $\mathrm{F}$ (two injection locations in the left pulvinar, seven and six sessions) and five sessions in monkey $\mathrm{R}$ (one injection location in the left pulvinar; Figure 1A). Because the results from the two injection sites in monkey $\mathrm{F}$ were similar, we pooled the data for the sake of clarity. Control (no inactivation) data collection was interleaved with drug injection sessions. Behavioral effects following THIP injections into the pulvinar typically lasted several hours. The minimum interval between injections was 2 days.

\section{Structural MRI Acquisition}

Anatomical images were acquired in a Bruker Biospec 4.7-T/60-cm vertical bore scanner equipped with a BGA38S2 gradient coil. A linear birdcage volume RF coil (Bruker; 
Figure 1. Inactivation sites and experimental design.

(A) Inactivation sites in the two monkeys visualized with coinjections of gadolinium MR contrast agent. The top shows coronal MR images (0.8-mm thickness) around the tip of the cannula between 20 and $40 \mathrm{~min}$ after THIP injection. The coinjected gadolinium appears white. In all cases, the center of the injection was between +3 and $+5 \mathrm{~mm}$ in Horseley-Clark coordinates. The bottom shows the estimated injection locations (orange). Estimation was performed by overlaying the pulvinar landmarks derived from the anatomical monkey atlas on the aligned and scaled MRI slices (Saleem \& Logothetis, 2006). Injections shown represent the typical injection volume used in the current study $(3-4 \mu \mathrm{l})$. Abbreviations: $\mathrm{bc}=$ brachium of SC; $c d=$ caudate; $\mathrm{PM}=$ medial pulvinar (target structure); $\mathrm{PL}=$ lateral pulvinar; $\mathrm{PI}=$ inferior pulvinar; $\mathrm{r}=$ reticular nucleus. (B) Memoryguided saccade task. A trial started with the presentation of a fixation spot followed by the

presentation of a saccade cue in either the left or right or in both hemifields, selected randomly from 18 possible positions. In instructed trials, only a single target was presented. In choice trials, two targets appeared simultaneously in the left and right hemifields equidistantly from the fixation point at the same vertical position. The color of the cue signaled the amount of upcoming reward (blue: low reward, green: high reward, red: low [equal] reward). Monkeys were trained on this cue-reward association before the start of the inactivation experiments. Monkeys were required to keep fixating following cue offset and to make a saccade following a 5 -sec memory delay. The offset of the fixation point served as signal to execute the saccade.

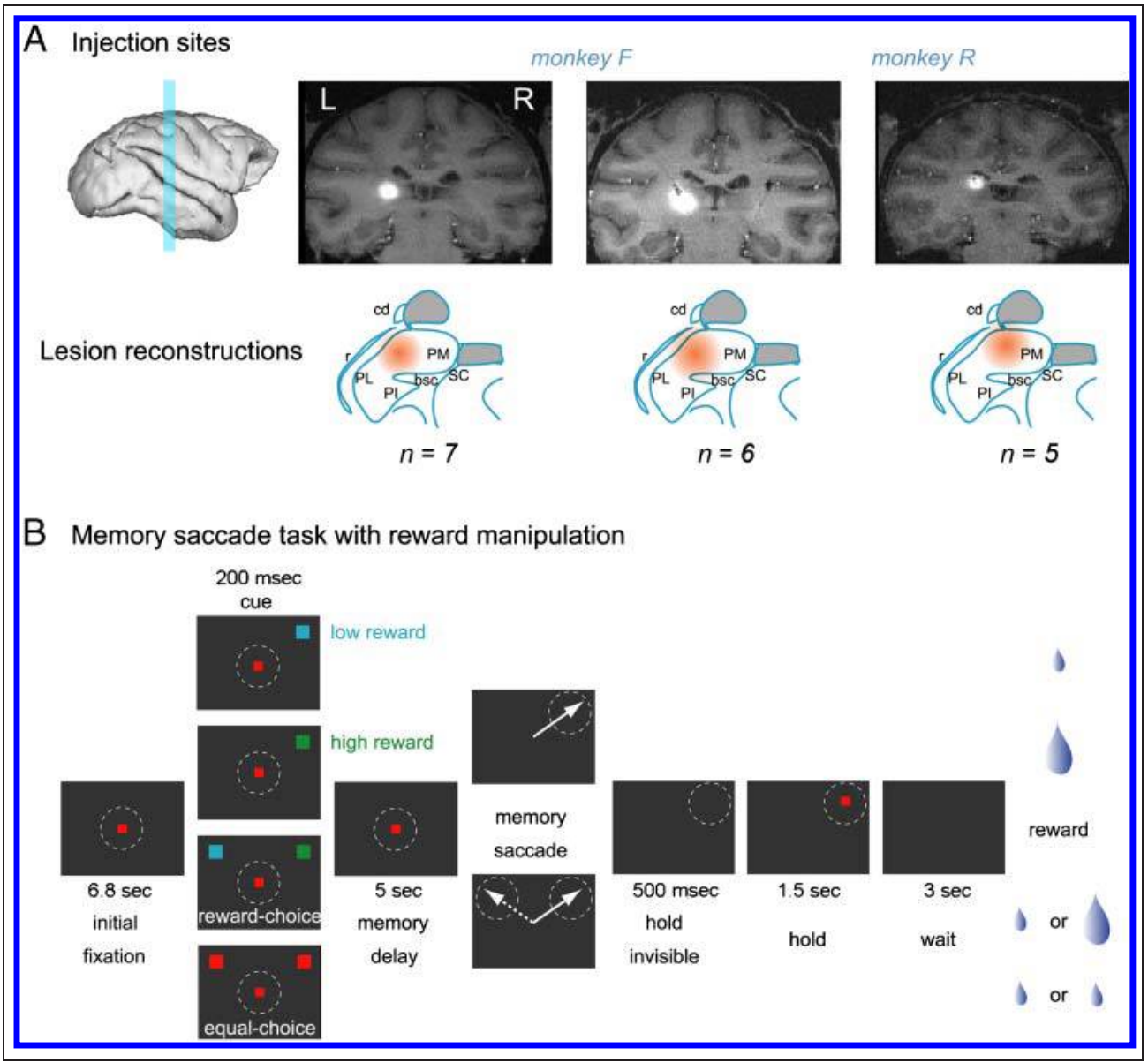

B Memory saccade task with reward manipulation monkeys $\mathrm{F}$ and R) or a quadrature surface RF coil (H. Merkle; monkey F), allowed whole-head coverage. Monkeys were previously adapted to the scanner environment and sat awake in a custom-made MR-compatible chair (Kagan, Iyer, Lindner, \& Andersen, 2010). Coronal anatomical T1-weighted images were acquired using a MDEFT sequence with an in-plane resolution of $0.5 \mathrm{~mm}$ and a slice thickness of $0.8 \mathrm{~mm}$.

\section{Behavioral Tasks}

Behavioral data were acquired between 50 and $180 \mathrm{~min}$ after the start of the injection.

\section{Memory-guided Saccade Task with Variable Reward Magnitude}

Monkeys were tested with a delayed memory saccade task consisting of two major trial types: (1) instructed trials, in which the presentation of a single cue defined the saccade target unequivocally; and (2) choice trials, in which two targets were presented in opposite hemifields equidistant from the central fixation, and monkeys could perform a saccade toward either one. The trial sequence is depicted in Figure 1B. Monkeys were required to fixate a red square in the middle of the screen. After $6.8 \mathrm{sec}$, either one (instructed) or two (choice) cues of $0.37^{\circ}$ square size were presented for $200 \mathrm{msec}$, indicating the target position(s) for the saccade and the associated reward magnitude. Following cue offset, monkeys were required to maintain the target position in memory and keep central fixation for another $5 \mathrm{sec}$ (memory period). The end of this memory delay period was signaled by the offset of the fixation spot ("go signal"), and the monkey had to perform a correct saccade within $500 \mathrm{msec}$. The saccade was judged to be correct if the eye position fell within a radius of $5^{\circ}$ and was held for additional $500 \mathrm{msec}$, after which the confirmation target at the previously cued position reappeared. The visual cues were randomly chosen from $18\left(10^{\circ}-16^{\circ}\right.$ eccentricity) locations, nine in the left and nine in the right visual hemifield. Incorrect trials with fixation breaks or incorrect saccades were 
aborted; successful trials were rewarded with water. Before the start of the experiments, monkeys were trained to associate the color of the cue with a certain reward magnitude that was delivered after a successful memory saccade (Figure 1B). Specifically, a blue cue predicted low reward, whereas a green cue predicted high reward (ratio: $1 / 3$, the liquid volumes ranged from $0.22-0.33 \mathrm{ml}$ for low reward to $0.66-0.99 \mathrm{ml}$ for high reward). Experiments started when monkeys selected the high reward cue in $>90 \%$ of the choice trials during training sessions. In instructed trials, the monkey had to perform a correct saccade even for low reward targets; otherwise, the trial with low reward magnitude was repeated until a correct response was made. Except for this low reward persistence, all trials types (target locations, instructed/choice, reward condition) were randomly interleaved.

\section{Memory-guided Saccade Task with Variable Contrast Levels}

To test for effects of visual saliency on saccade performance and choices, we varied in a subset of sessions the luminance contrast of the saccade cues (10\% vs. 100\% of the maximal red luminance presented on the black background) while keeping the reward amount constant. Although we could not measure the absolute luminance of the stimuli because we used the LCD goggles with the incorporated lens optics for stimulus presentation, the low contrast cue was faint but suprathreshold for goggle-adapted humans in the dark room (similar to the scanner bore). All other aspects of the task were the same as described for the reward experiments above. In the six control and inactivation sessions where we tested the effect of luminance contrast, those were interleaved with the reward magnitude experiment in a counterbalanced block-wise manner, with each block having a duration of 20 min.

\section{Stimulus Presentation, Online Behavioral Control, and Data Acquisition}

Visual stimuli were presented on $800 \times 600$, 60-Hz LCD goggles (Resonance Technology, Northridge, CA) subtending $30^{\circ} \times 24^{\circ}$ of visual angle using custom OpenGL software. Eye position was monitored at $60 \mathrm{~Hz}$ and $0.5^{\circ}-1^{\circ}$ accuracy with a mini-IR camera (Resonance Technology or Arrington Research, Scottsdale, AZ) and recorded together with stimulus and timing information. Online behavioral control and feedback were implemented in a LabVIEW RT (National Instruments, Austin, TX).

\section{Eye Movement Recording and Analysis}

The position of the eye was monitored in real time and stored off-line. Trials in which the monkey did not maintain fixation or exceeded the saccade reaction time criteria (500 msec from the offset of the fixation point) were aborted. The central fixation window radius was $3^{\circ}-4^{\circ}$, and the peripheral saccadic target window radius was $5^{\circ}$. At $60-\mathrm{Hz}$ sampling rate of the video-based IR eye tracker, saccades of $>0.33^{\circ}$ amplitude can in principle be detected by a $20^{\circ} / \mathrm{sec}$ velocity threshold. However, because of instrumental noise in the eye tracker, resulting in $\sim 0.5^{\circ}$ accuracy, we limited our analysis to saccades of $>1^{\circ}$ that could be reliably identified using a custom combined velocity and acceleration algorithm.

Preliminary data analysis did not reveal a systematic effect of pulvinar inactivation on particular target positions within a given hemifield. Thus, results for the different locations within a given hemifield were pooled together. Unless otherwise specified, data were submitted to a three-way ANOVA with the factors Drug (control vs. inactivation), Reward (low vs. high), and Hemifield (left vs. right). This analysis was followed by a two-tailed $t$ test for statistical comparisons separately for each hemifield. The significance level was set to $p<.05$.

\section{RESULTS}

We tested two monkeys following injection of the GABAA agonist THIP into the dorsal pulvinar (13 sessions in monkey $\mathrm{F}, 5$ sessions in monkey R, and the same number of interleaved control sessions). Injection locations were initially planned by means of presurgical anatomical MRI and verified in each session by imaging the spread of the coinjected contrast agent gadolinium associated with the injection (Experimental Methods). Imaging data indicate that inactivation was primarily in the medio-dorsal pulvinar (Figure 1A). We use the terms "ipsilesional" and "contralesional" with respect to the inactivated hemisphere (e.g., after an injection into the left hemisphere, the left visual hemifield is "ipsilesional").

\section{Inactivation Effects on Memory Saccades with Different Rewards: Instructed Trials}

To examine the effect of pulvinar inactivation on saccades toward memorized targets, we employed a delayed memory saccade task with single targets (Figure 1B, "instructed"). Targets were associated with different reward magnitudes, which were signaled by the color of the cue. Monkeys were trained on this association before the beginning of the experiments. We evaluated two different types of saccade errors: (1) fixation break saccades (premature saccade before the go signal but after the cue was presented) and (2) incorrect saccades (saccade after the go signal but to the wrong position). After inactivation, there was no substantial impairment in the monkey's ability to correctly remember the cued target position and saccade toward it (Figure 2A). In control sessions, there was a tendency for fixation aborts toward cues signaling higher reward, which reached significance for the right hemifield in monkey $\mathrm{F}$ and for both hemifields in monkey $\mathrm{R}$ (two-tailed $t$ test, $p<.05$; Figure 2B, Table 1 ). In addition, the proportion of correctly executed saccades after the delay period 


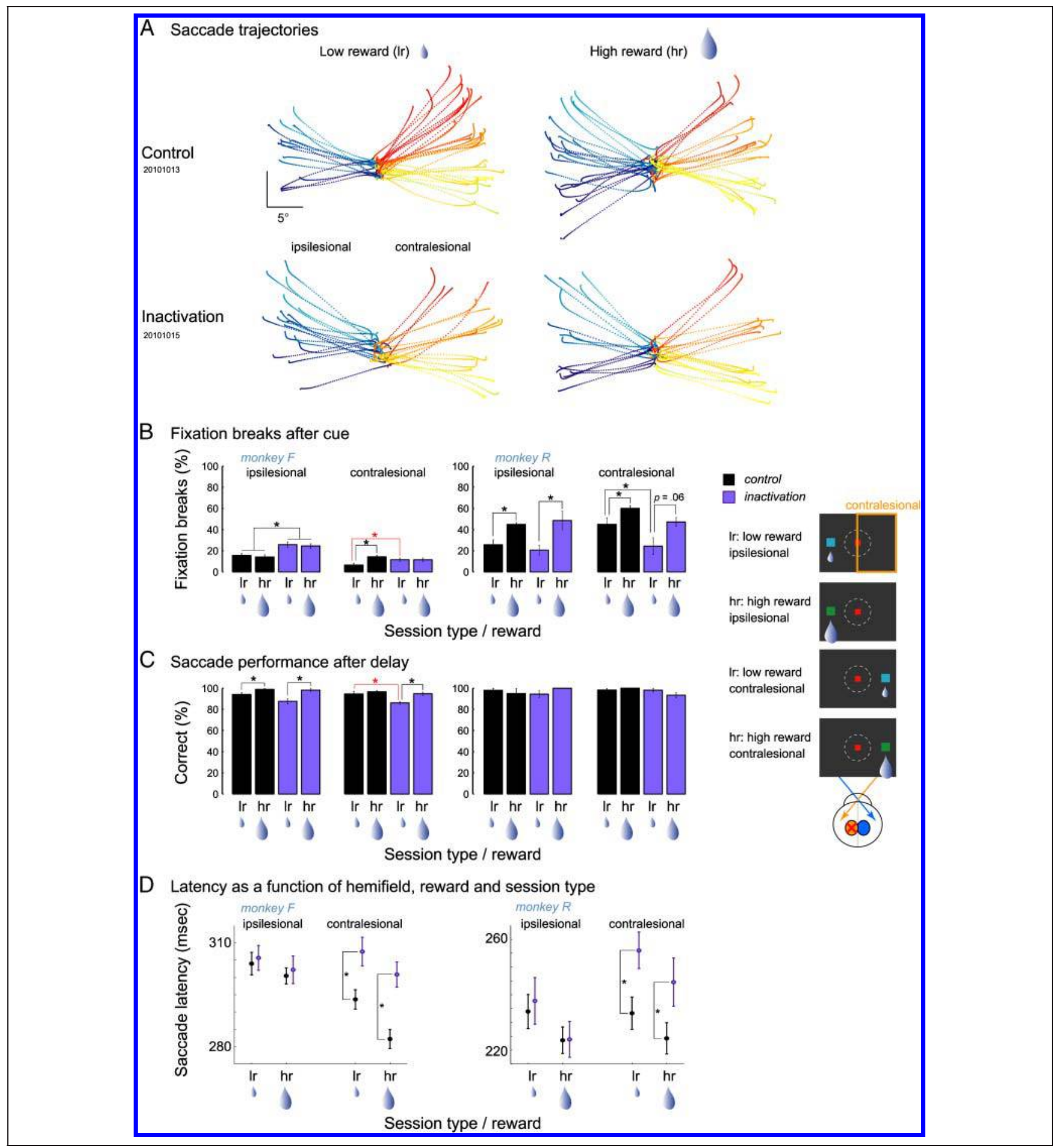

Figure 2. Instructed saccades. (A) Trajectories of instructed memory saccades toward single targets in a typical control and inactivation session in monkey F. Saccades were made to targets in 18 possible directions at an eccentricity between $10^{\circ}$ and $16^{\circ}$. Saccadic eye movements (dotted curves) in control (top) and inactivation sessions (bottom) for low (left) and high (right) reward. Trajectory colors denote the locations of the saccade targets (red-yellow: right, cyan-blue: left; scale bar, $5^{\circ}$ ). Here, and in all other figures, the left hemifield is ipsilesional, and right hemifield is contralesional after the inactivation. (B) Effect of inactivation on fixation aborts. The percent of fixation aborts following cue presentation for each hemifield, reward condition, and monkey. Data were averaged over sessions and pooled over the nine target positions in each hemifield. Here and in other figures, purple bars represent data obtained after inactivation; black bars, data from control sessions. Error bars indicate $S E$ across sessions, and stars denote $p<.05$ for the specific comparison as indicated by connecting lines (red asterisk denotes contralesional deficits). Inset on the right illustrates the four instructed cue conditions. (C) Effect of inactivation on saccade performance. The percent of correct saccades that monkeys made after they successfully maintained fixation during the delay period for each hemifield, reward condition, and monkey. Same notations as in (B). Red star indicates the contralesional decrease of correct low reward saccades in monkey F, the only contralesional deficit besides the prolongation of saccade latencies observed for the instructed trials. (D) Mean saccade latencies as a function of reward and hemifield. Saccade latencies toward the contralesional space became longer in both monkeys after inactivation. 
Table 1. Task Performance in Instructed Trials for Control and Inactivation Sessions

\begin{tabular}{|c|c|c|c|c|c|c|c|c|c|c|}
\hline \multirow[b]{2}{*}{ Monkey } & \multirow[b]{2}{*}{ Session } & \multirow[b]{2}{*}{$H F$} & \multicolumn{2}{|c|}{ Fixation Breaks (\%) } & \multicolumn{2}{|c|}{ Saccade Errors (\%) } & \multicolumn{2}{|c|}{$\begin{array}{c}\text { Overall } \\
\text { Performance (\%) }\end{array}$} & \multicolumn{2}{|c|}{ Latency (msec) } \\
\hline & & & $\begin{array}{c}\text { Low } \\
\text { Reward }\end{array}$ & $\begin{array}{c}\text { High } \\
\text { Reward }\end{array}$ & $\begin{array}{c}\text { Low } \\
\text { Reward }\end{array}$ & $\begin{array}{c}\text { High } \\
\text { Reward }\end{array}$ & $\begin{array}{c}\text { Low } \\
\text { Reward }\end{array}$ & $\begin{array}{c}\text { High } \\
\text { Reward }\end{array}$ & $\begin{array}{c}\text { Low } \\
\text { Reward }\end{array}$ & $\begin{array}{c}\text { High } \\
\text { Reward }\end{array}$ \\
\hline \multirow[t]{4}{*}{$\mathrm{F}$} & Control & $\mathrm{LH}$ & $15.6 *$ & 14.1* & $5.9 * *$ & $1.0 * *$ & $79.5 * * *$ & $85.1 * * *$ & 304 & 300 \\
\hline & Inactivation & LH & $25.8^{*}$ & $24.5 *$ & $12.4 * *$ & $1.8 * *$ & $65.5 *$ & $74.1 *$ & 306 & 302 \\
\hline & Control & $\mathrm{RH}$ & $6.5^{*} * *$ & $14.2 * *$ & $5.4^{*}$ & 3.3 & $88.5^{*}$ & 82.9 & $294 * * *$ & $282 *$ \\
\hline & Inactivation & RH & 11.6* & 11.5 & $13.9 * * *$ & $5.2 * *$ & $76.2 * * *$ & $83.9 * *$ & $307 *$ & $301 *$ \\
\hline \multirow[t]{4}{*}{$\mathrm{R}$} & Control & LH & $25.5^{* *}$ & $44.9 * *$ & 2.0 & 5.0 & $72.8 * *$ & $52.6 * *$ & 234 & 224 \\
\hline & Inactivation & LH & 20.1 & 48.6 & 1.7 & 0 & 74.5 & 51.5 & 238 & 224 \\
\hline & Control & RH & $45.0 * * *$ & $60.0 * *$ & 1.7 & 0 & $53.8 * * *$ & $39.9 * *$ & $233^{*}$ & $224 *$ \\
\hline & Inactivation & RH & $24.4^{*}$ & 47.3 & 1.82 & 6.5 & $74.8^{*}$ & 49.3 & $256^{*}$ & $245^{*}$ \\
\hline
\end{tabular}

LH = left (ipsilesional) hemifield; RH = right (contralesional) hemifield. Significant inactivation effects are in bold.

$* p<.05$ for comparison between control versus inactivation (separate two-tailed $t$ tests within each reward condition and hemifield).

$* * p<.05$ for comparison between low reward versus high reward (separate two-tailed $t$ tests within session and hemifield).

was significantly increased in monkey $\mathrm{F}$ for high rewarded positions, and saccade latencies tended to be shorter in those trials in both monkeys (Figure 2C, Table 1).

In both monkeys, inactivation affected the pattern of fixation aborts, as indicated by an interaction between the factors Drug $\times$ Hemifield (ANOVA, $p<.05$ in monkey $\mathrm{F}$, $p=.06$ in monkey R). Specifically, monkey $\mathrm{F}$ showed a significant increase of fixation aborts toward cues in the ipsilesional hemifield, whereas fixation aborts toward the contralesional hemifield decreased following inactivation in monkey R (Figure 2B, Table 1). The only significant contralesional impairments in instructed trials were the increased fixation breaks and slightly decreased saccade performance for low-reward contralesional targets in monkey F (Figure 2B, red asterisk).

The most pronounced and consistent effect of inactivation on instructed trials was observed for saccade latencies. In both monkeys, inactivation resulted in a significant increase of saccade latencies for contralesional positions (ANOVA, Drug $\times$ Hemifield, $p<.05$; Figure 2D). Separate analysis for the ipsilesional and contralesional hemifields revealed a significant effect of inactivation for both lowand high-reward contralesional targets (two-tailed $t$ test, $p<.05)$ but not for ipsilesional targets $(p>.7$; Table 1$)$. No significant interaction between Drug $\times$ Reward or Drug $\times$ Reward $\times$ Hemifield was found in instructed trials for any performance measure or saccade latencies, further indicating that pulvinar inactivation did not interfere with reward processing per se (Figure 2A-D).

\section{Effects on Saccade Choices between Equal and Unequal Options}

To investigate how pulvinar inactivation altered selection between two response options, we next examined the inactivation effects on choices between two simultaneously presented cues in both hemifields. The two cues predicted either equal or differing reward magnitudes, as indicated by their color (Figure 1B, "choice"). Although individual spatial preferences were apparent in control sessions (monkey F preferred right hemifield, and monkey R preferred left hemifield under conditions of equal reward), monkeys still chose both sides of space when the two cues did not differ in respect to expected reward magnitude (Figure 3A and B). Following inactivation, monkeys showed a strong decrease of contralesional choices (Figure $3 \mathrm{~A}$ and $\mathrm{B}$ ) when selecting between equal rewards. Specifically, monkey $\mathrm{F}$ chose right (contralesional to be) targets in $78 \%$ of the trials in control sessions; equal reward choices toward the contralesional field decreased to $42 \%$ following pulvinar inactivation (one-way ANOVA, main effect of drug, $p<.05$ ). A similar effect was found in monkey $\mathrm{R}$ (control: $31 \%$ vs. inactivation: $5 \%, p<.05$; Figure 3B).

We next evaluated the effect of reward expectation on choices between targets with unequal reward magnitude. As predicted from previous studies (McCoy \& Platt, 2005; Platt \& Glimcher, 1999), in control sessions, monkeys typically selected the target that predicted the higher reward $(>95 \%)$. For the inactivation sessions, we hypothesized that perceptual extinction during bilateral cue presentation would be expressed as a tendency to choose ipsilesional targets even if they yielded the low reward. On the other hand, if pulvinar inactivation does not act primarily on the perceptual processing but, for instance, leads to an increased effort to perform a contralesional movement (thus decreasing "desirability" of contralesional space), we expected that a higher reward magnitude for contralesional positions will increase the proportion of choices toward this side. The pattern of results supports the latter hypothesis. After inactivation, monkeys still 


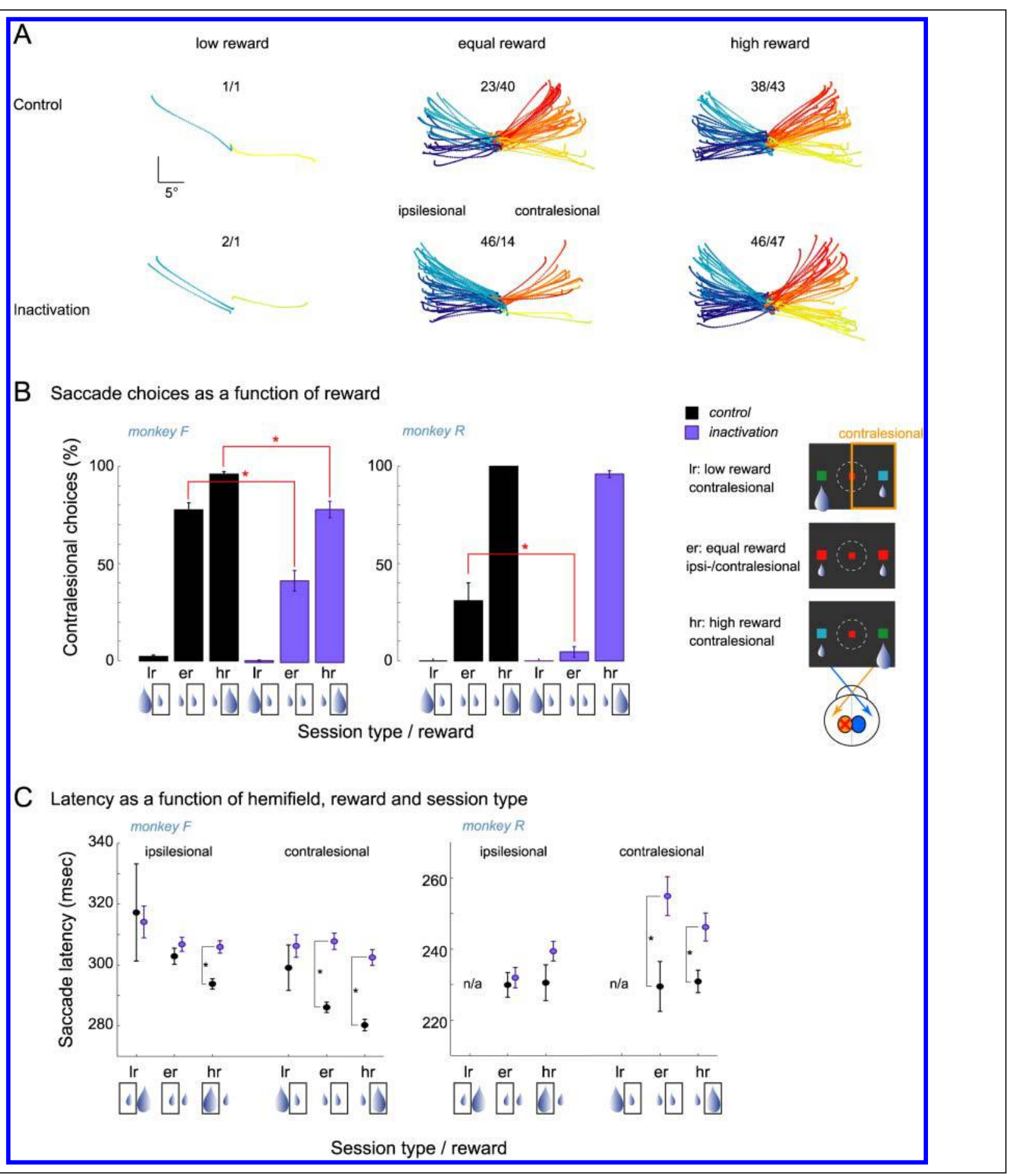

Figure 3. Choice behavior. (A) Trajectories of selected memory saccades in choice trials in a typical control and inactivation session in monkey $\mathrm{F}$ (same sessions as in Figure 2A for instructed saccades). Note the inactivation-induced decrease of choices toward the contralesional space in the equal reward condition as opposed to the unaffected contralesional choice behavior for targets associated with high reward. The numbers above the trajectories indicate the number of saccades made to the left and right hemifields. Other notations as in Figure 2A. (B) Percentage of saccades toward the contralesional (right) hemifield in choice trials as a function of reward and session type. In both monkeys, the percentage of contralesional choices decreased significantly during selection between equal rewards but remained high when a large reward was placed in the contralesional hemifield. Inset on the right illustrates the three-choice cue conditions. The rectangular frame around the small or large reward icon in each left/right reward pair under the horizontal axis denotes the corresponding selection. (C) Saccade latencies for ipsilesional and contralesional choices as a function of expected reward. Latencies for low reward choices were not plotted in monkey R because of the lack of those trials. Note that saccade latencies toward the contralesional space became longer in both monkeys after inactivation, similar to instructed saccades. 
strongly preferred contralesional over ipsilesional target positions when the contralesional cue indicated the higher reward (monkey $\mathrm{F}$ : control $=96 \%$ vs. inactivation $=79 \%$; monkey R: control $=100 \%$ vs. inactivation $=96 \%$ ). Thus, although inactivation somewhat reduced the proportion of contralesional high-reward choices (ANOVA for highreward choices, main effect of Drug: monkey $\mathrm{F}, p<$ .05 ; monkey $\mathrm{R}, p=.09$ ), reward expectation largely overcame the inactivation-induced spatial choice bias in both monkeys.

However, despite almost complete alleviation of the choice bias by the high reward, we still observed increased latencies to contralesional low- and high-reward targets, as in instructed saccades (Figure 3C, Table 2). Accordingly, a separate analysis for each hemifield revealed a significant inactivation effect for contralesional saccades in both monkeys (two-tailed $t$ test, monkeys $\mathrm{F}$ and $\mathrm{R}, p<.05)$. Although the latency increase after inactivation was more pronounced for contralesional targets, monkey $\mathrm{F}$ also showed a latency increase for ipsilesional high-reward saccades (monkey F, $p<.05$; monkey $\mathrm{R}$, $p=.37$ ). Taken together, this pattern of results indicates at least a partial dissociation between response selection and saccade initiation deficits.

\section{Effect of Visual Saliency on Saccade Choices}

The alleviation of the spatial choice bias by high reward association suggested that the bias following pulvinar inactivation in the equal reward condition was not caused by a perceptual deficit but rather by an attentional/intentional impairment. Nonetheless, we wondered whether monkeys overcame their choice bias because the cue associated with the high reward value had acquired a higher perceptual saliency. To assess the influence of visual saliency on choice behavior more directly, we conducted six control and six inactivation sessions in monkey $\mathrm{F}$, where we directly varied the luminance of the cues, without reward manipulation. The temporal structure of the task was as in the reward experiments described above (Figure 1B). In instructed trials, a single target of either low (10\%) or high luminance (100\% of the luminance used in the regular equal reward condition) was shown, and in choice trials, the two targets, presented simultaneously in both hemifields, were either of equal or differing luminance (Figure 4A, right inset). As illustrated in Figure 4A, pulvinar inactivation significantly increased the number of fixation aborts (ANOVA, main effect of Drug, $p<.05$; Table 3). Fixation aborts after inactivation were more pronounced toward the ipsilesional hemifield, although this tendency was also present for contralesional positions (ANOVA, Hemifield $\times$ Drug, $p=.49$ ). Saccade errors were not significantly affected in either hemifield (main effect of Drug, $p=.09$; Hemifield $\times$ Drug, $p=.07$; Figure $4 \mathrm{~B})$. No significant interaction between Contrast $\times$ Drug or Contrast $\times$ Drug $\times$ Hemifield was found for either error type $(p>.4)$, indicating that pulvinar lesions did not specifically impair processing of low luminance saccade cues (Figure 4A and B). Inactivation increased saccade latencies for both ipsilesional and contralesional stimuli, although this comparison did not reach statistical significance $(p>.07$; Figure $4 \mathrm{C})$.

Most relevant for our question is the analysis of choices between targets with equal or different luminance contrasts (Figure 4D, left). As in the reward experiment described above, inactivation significantly decreased the proportion of contralesional choices when the luminance in both hemifields was equal (control: $72 \%$ vs. inactivation: 22\%; one-way ANOVA, main effect of Drug, $p<.05)$. In control sessions, when given the choice between two

Table 2. Saccade Latencies in Choice Trials for Control and Inactivation Sessions

\begin{tabular}{|c|c|c|c|c|c|}
\hline \multirow[b]{2}{*}{ Monkey } & \multirow[b]{2}{*}{ Session } & \multirow[b]{2}{*}{$H F$} & \multicolumn{3}{|c|}{ Latency (msec) } \\
\hline & & & Low Reward & Equal Reward & High Reward \\
\hline \multirow[t]{4}{*}{$\mathrm{F}$} & Control & $\mathrm{LH}$ & $317^{*}$ & 303 & $294 * * *$ \\
\hline & Inactivation & $\mathrm{LH}$ & 314 & 307 & $306 * *$ \\
\hline & Control & $\mathrm{RH}$ & 299 & $287 * *$ & $280 * *$ \\
\hline & Inactivation & $\mathrm{RH}$ & 306 & $308 * *$ & $302 * *$ \\
\hline \multirow[t]{4}{*}{$\mathrm{R}$} & Control & $\mathrm{LH}$ & n.a. & 231 & 232 \\
\hline & Inactivation & $\mathrm{LH}$ & n.a. & 233 & 240 \\
\hline & Control & $\mathrm{RH}$ & n.a. & 231 & $232 * *$ \\
\hline & Inactivation & $\mathrm{RH}$ & n.a. & 256 & $247 * *$ \\
\hline
\end{tabular}

n.a. $=$ not applicable because of lack of trials.

*Next to the equal (low) reward value denotes $p<.05$ for comparison between equal (low) reward versus high reward (separate two-tailed $t$ tests for within session and hemifield).

**Next to the control value denotes $p<.05$ for comparison between control versus inactivation (separate two-tailed $t$ tests for within each reward condition and session type). 


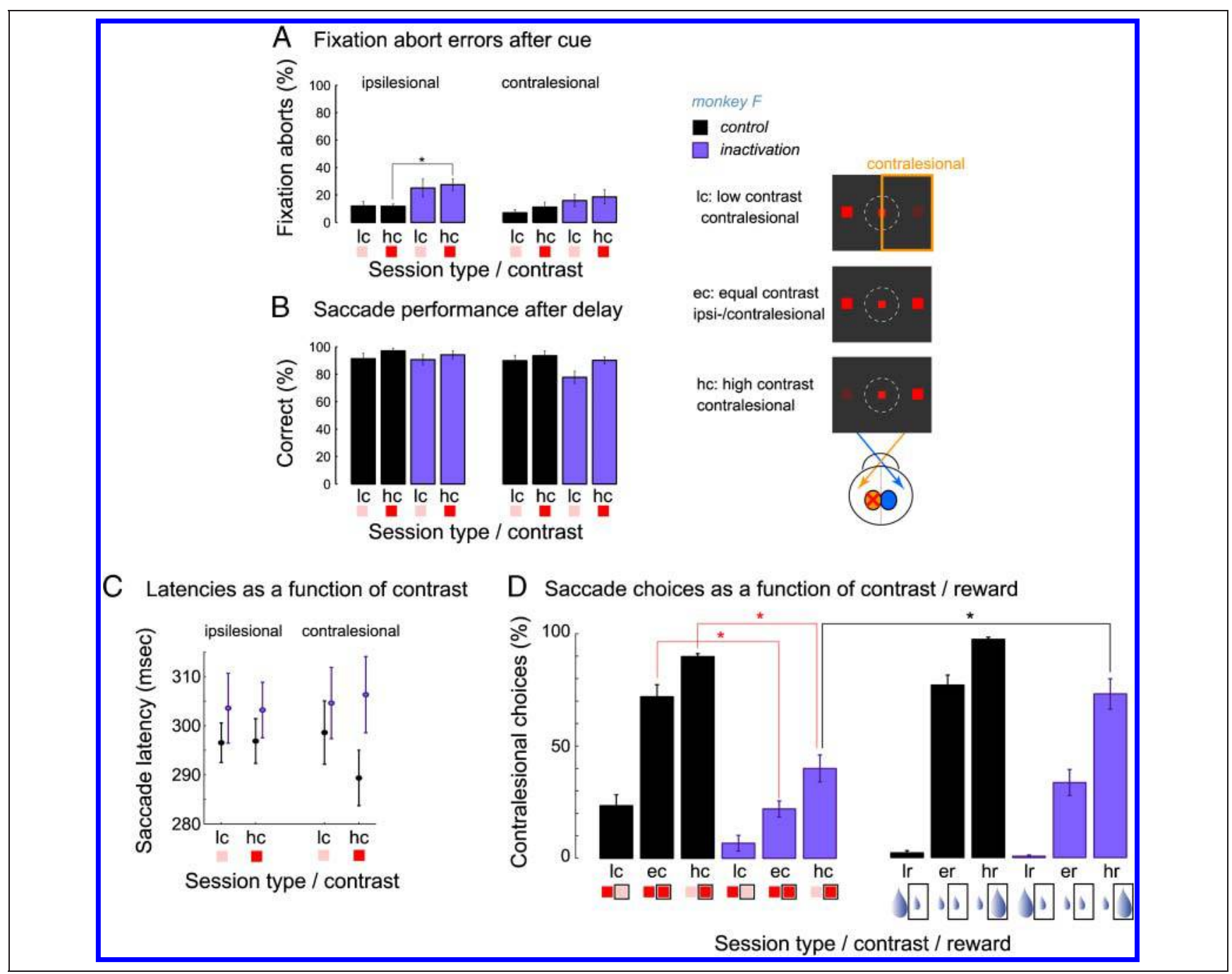

Figure 4. Effect of luminance contrast on saccade behavior and choices. Memory-guided saccade task with luminance contrast manipulation (monkey F, six control and six inactivation sessions). The general task structure was as described in Figure 1B. The luminance contrast of the targets was 10\% (low contrast) or 100\% (high contrast). Positions of low and high contrast cues were randomly varied between 18 possible target positions. Reward magnitude was held constant for all contrast levels. Inset on the right illustrates the three-choice cue conditions (single-target instructed conditions are not shown). (A) The percent of fixation aborts in instructed trials following cue presentation for each hemifield and contrast condition. (B) Proportion of correct saccades in instructed trials for each hemifield and contrast condition. (C) Mean saccade latency in instructed trials as a function of contrast and hemifield. (D, left) Percentage of saccades toward the contralesional (right) hemifield in choice trials as a function of contrast and session type, in contrast manipulation blocks. Note that, after inactivation, even when a high contrast target was presented in the contralesional field together with a low contrast target in the ipsilesional field, the monkey still more often selected the ipsilesional target (60\%). The rectangular frame around the small (faint) or large (saturated) contrast icon in each left/right contrast pair under the horizontal axis denotes the corresponding selection. (D, right) Percentage of saccades toward the contralesional (right) hemifield in choice trials as a function of reward and session type, in reward manipulation blocks, tested in the same sessions with contrast manipulation blocks. These data are a subset of 13 control and 13 inactivation reward manipulation sessions shown in Figure 3B for monkey F.

targets with different luminance, the monkey preferred the high over the low luminance targets ( $90 \%$ choices of high luminance in the right, contralesional hemifield to be; and $77 \%$ in the left hemifield). Following inactivation, a high luminance target in the contralesional hemifield coupled with a low luminance target in the ipsilesional hemifield somewhat increased the proportion of contralesional choices as compared with the equal luminance condition ( $40 \%$ vs. $22 \%$, respectively). Nonetheless, as described in the previous section, a high reward stimulus alleviated the ipsilesional selection bias more effectively than manipulation of visual saliency (monkey F, highreward contralesional selection of $79 \%$ overall). In the subset of six inactivation sessions in monkey $\mathrm{F}$ where we interleaved both the reward and luminance contrast manipulations, contralesional choice was $70 \%$ for high reward vs. $40 \%$ for high contrast, which was significantly different (Figure 4D, right; Table 4).

The higher effectiveness of reward as opposed to the luminance manipulation is also expressed by a significant 
Table 3. Task Performance in Instructed Trials in Control and Inactivation Sessions, for Low and High Contrast Trials

\begin{tabular}{|c|c|c|c|c|c|c|c|c|c|c|}
\hline \multirow[b]{2}{*}{ Monkey } & \multirow[b]{2}{*}{ Session } & \multirow[b]{2}{*}{$H F$} & \multicolumn{2}{|c|}{ Fixation Breaks (\%) } & \multicolumn{2}{|c|}{ Saccade Errors (\%) } & \multicolumn{2}{|c|}{$\begin{array}{c}\text { Overall } \\
\text { Performance (\%) }\end{array}$} & \multicolumn{2}{|c|}{ Latency (msec) } \\
\hline & & & $\begin{array}{c}\text { Low } \\
\text { Contrast }\end{array}$ & $\begin{array}{c}\text { High } \\
\text { Contrast }\end{array}$ & $\begin{array}{c}\text { Low } \\
\text { Contrast }\end{array}$ & $\begin{array}{c}\text { High } \\
\text { Contrast }\end{array}$ & $\begin{array}{c}\text { Low } \\
\text { Contrast }\end{array}$ & $\begin{array}{c}\text { High } \\
\text { Contrast }\end{array}$ & $\begin{array}{c}\text { Low } \\
\text { Contrast }\end{array}$ & $\begin{array}{c}\text { High } \\
\text { Contrast }\end{array}$ \\
\hline \multirow[t]{4}{*}{$\mathrm{F}$} & Control & $\mathrm{LH}$ & 11.9 & $11.8^{*}$ & 8.6 & 2.9 & 80.2 & $85.6 *$ & 297 & 297 \\
\hline & Inactivation & $\mathrm{LH}$ & 25.1 & $27.5 *$ & 9.4 & 5.8 & 67.4 & $68.2 *$ & 303 & 303 \\
\hline & Control & $\mathrm{RH}$ & 7.0 & 11.2 & 10.1 & 6.5 & $83.5 *$ & 83.6 & 299 & 289 \\
\hline & Inactivation & RH & 15.9 & 18.6 & 22.2 & 9.9 & $64.8 *$ & 73.82 & 304 & 306 \\
\hline
\end{tabular}

Significant inactivation effects are printed in bold. None of the comparisons between low contrast versus high contrast reached significance (separate two-tailed $t$ tests for within session and hemifield).

$* p<.05$ for comparison between control versus inactivation (separate two-tailed $t$ tests within each contrast condition and session type)

interaction in the ANOVA between Drug $\times$ "Salience manipulation" (i.e., reward vs. luminance; $p<.05$ ).

\section{DISCUSSION}

Lesions in the dorsal pulvinar lead to spatial deficits such as an ipsilesional oculomotor choice bias. In this study, we tested whether pulvinar inactivation affects saccades toward memorized spatial positions and reward-based target selection. We were particularly interested to see whether the inactivation-induced spatial choice bias could be overcome by manipulation of cue-associated reward values. Although pulvinar inactivation increased memory saccade latencies for contralesional targets, it did not impair the ability to perform correct memory saccades. Most importantly, we found that the strong ipsilesional choice bias that was present under equal reward conditions was strongly reduced when the cue in the contralesional field predicted the high reward.

\section{Pulvinar and Oculomotor Behavior}

Inactivation resulted in an increase of memory saccade latencies toward contralesional targets. Such an increase

Table 4. Percent of Right Hemifield (Contralesional) Choices for Six Control and Six Inactivation Sessions as a Function of Saliency Manipulation

\begin{tabular}{|c|c|c|c|c|c|c|}
\hline \multirow{3}{*}{$\begin{array}{l}\text { Salience } \\
\text { Manipulation }\end{array}$} & \multicolumn{6}{|c|}{ Choices Towards RH (\%) } \\
\hline & \multicolumn{3}{|c|}{ Control } & \multicolumn{3}{|c|}{ Inactivation } \\
\hline & Low & Equal & High & Low & Equal & High \\
\hline Reward & 2 & 77 & 97 & 1 & 33 & 70 \\
\hline Luminance & 23 & 72 & 90 & 7 & 22 & 40 \\
\hline
\end{tabular}

Reward and luminance manipulations were conducted in control and inactivation sessions in interleaved counterbalanced blocks within each session. Significant effects of manipulation in inactivation sessions are printed in bold. for contralesional positions was not found in a previous study that employed a speeded visually guided saccade task (Wilke, Turchi, et al., 2010). It is conceivable that pulvinar inactivation results in an intentional movement deficit, which is more pronounced when no visual stimulus is present. The dorsal pulvinar is reciprocally interconnected with several areas in the parietofrontal cortex such as the lateral intraparietal (LIP) area, FEF, and the dorsolateral pFC (dlPFC), where electrophysiological studies in monkeys have reported firing rate changes during cue and delay-period intervals in the context of memory saccade tasks (Takeda \& Funahashi, 2002; Chafee \& Goldman-Rakic, 1998; Gnadt \& Andersen, 1988). Correspondingly, several studies demonstrated that lesions in these areas impair performance in memory saccade tasks (Wilke, Kagan, \& Andersen, 2012; Liu, Yttri, \& Snyder, 2010; Dias \& Segraves, 1999; Li, Mazzoni, \& Andersen, 1999; Funahashi, Bruce, \& Goldman-Rakic, 1993). These studies revealed that LIP and FEF inactivation were associated with longer latencies and hypometric saccades toward contralesional positions (Dias \& Segraves, 1999; Li et al., 1999), whereas dlPFC lesions did not result in longer latencies but were characterized by erroneous saccade directions (Funahashi et al., 1993). Thus, our findings are consistent with the view that dorsal pulvinar lesions lead to increased memory saccade latencies by affecting activity within and/or between LIP and FEF areas. At the same time, one of the monkeys showed an increase of fixation aborts toward single ipsilesional cues after pulvinar inactivation. This impairment of saccade inhibition is reminiscent of the higher number of ipsilesional express saccades following pulvinar inactivation that has been reported in a previous study that used a direct saccade paradigm (Wilke, Turchi, et al., 2010). Nonetheless, the effects of pulvinar inactivation on saccade performance toward single targets were modest overall, and we thus conclude that the medio-dorsal pulvinar is less critical for perceptual and mnemonic processes but is more important for the formation of movement intentions such as saccade planning and initiation. 


\section{Pulvinar and Equal Reward Choices}

We found that pulvinar inactivation led to a strong reduction of contralesional memory-guided saccade choices if a saccade toward the ipsilesional space yielded the same amount of reward. This choice bias is consistent with a previous inactivation study that employed a visually guided saccade task in monkeys (Wilke, Turchi, et al., 2010) and also corresponds to studies in humans with pulvinar lesions (Arend, Machado, et al., 2008). Although we cannot rule out the possibility of stochastic perceptual extinction, monkeys typically did choose the contralesional target when the cue color signaled the higher reward (see below), and we thus interpret this bias not as primary detection deficit but as movement intention deficit.

One possibility is that pulvinar inactivation increased the thresholds to trigger a saccade toward the contralesional space, whereas the thresholds for positions in the ipsilesional space either decreased or remained the same. This interpretation is consistent with the finding that pulvinar inactivation resulted in longer memory saccade latencies for contralesional targets even in the absence of ipsilesional competition. Because the dorsal pulvinar exchanges reciprocal connections with fronto-parietal cortical areas and area STP in the dorsal bank of the STS, which support visuospatial attention and eye movement behavior, those deficits could be interpreted as dysfunction in those thalamo-cortical networks (Saalmann \& Kastner, 2011; Kaas \& Lyon, 2007; Sherman, 2005; Gutierrez et al., 2000; Scalaidhe, Albright, Rodman, \& Gross, 1995; Blatt, Andersen, \& Stoner, 1990). Thus, by applying the interhemispheric imbalance model (Kinsbourne, 1977), one could assume that the latency increase and the reduced choices of contralesional targets are because of hypoactivity and/or disruption of cortico-cortical communication in those areas within the lesioned hemisphere. This interpretation is particularly plausible with regard to previous findings of similar choice bias following lesions in LIP (Wilke et al., 2012; Wardak, Olivier, \& Duhamel, 2002) and FEF (Schiller \& Chou, 1998). In addition, hyperactivity in response to ipsilesional targets in corresponding areas within the intact hemisphere could also contribute to the observed ipsilesional choice bias.

\section{Pulvinar and Reward-based Choices}

Consistent with previous work in intact animals, when reward magnitude differed between the two possible choices, monkeys strongly preferred the position that predicted the higher reward (McCoy \& Platt, 2005; Opris \& Bruce, 2005). Importantly, this choice pattern remained almost intact following inactivation, showing that the spatial bias could be overcome by manipulating the reward associated with the contralesional target. One interpretation can be that the dorsal pulvinar inactivation does not impair the perceptual visibility of contralesional targets but instead affects the intentional, motivational encoding of contralesional movements. The high reward may have increased the willingness of the monkeys to exert additional effort. This is in line with human and animal studies showing that action decisions are weighted by reward and the required effort (Kurniawan, Guitart-Masip, \& Dolan, 2011). Also consistent with increased effort to overcome the behavioral extinction are recent findings in a study in which a decision bias was produced by unilateral reversible inactivation of LIP in monkeys (Wilke et al., 2012). In trials in which the animals still chose targets in the contralesional hemifield, visual-oculomotor cortical areas in both hemispheres showed increased BOLD signals, suggesting bihemispheric compensation.

Studies in patients with cortical lesions have shown that emotional and motivational factors have an impact on neglect and extinction behavior (Dominguez-Borras, Saj, Armony, \& Vuilleumier, 2012). For example, preliminary reports indicated that reward-predicting stimuli such as money symbols can improve the detection performance of neglect patients for contralesional targets (Lucas, Diserens, Leroy, Krattinger, \& Vuilleumier, 2005; Mesulam, 1999), and it has been demonstrated that extinction symptoms can be improved by adding emotional content to the stimuli such as presenting angry or happy faces (Fox, 2002; Vuilleumier et al., 2002; Vuilleumier \& Schwartz, 2001), emotional scenes (Grabowska et al., 2011), or pleasant music (Soto et al., 2009). Likewise, studies in healthy participants indicate that food and money incentives improve spatial attention (Padmala \& Pessoa, 2010; Engelmann \& Pessoa, 2007) and perceptual processing of reward-associated stimulus features (Hickey, Chelazzi, \& Theeuwes, 2010; Pleger, Blankenburg, Ruff, Driver, \& Dolan, 2008). The effects of stimulus-reward association in the current study are in agreement with this literature.

Was the selection of high-reward targets driven by their potentially higher perceptual visibility? The exact nature of the interaction between reward and attentional/perceptual processes remains unresolved (Pessoa \& Engelmann, 2010), and a recent fMRI study demonstrated considerable overlap between neural networks involved in cueassociated reward processing and attention (Krebs, Boehler, Roberts, Song, \& Woldorff, 2012). Thus, although one may attempt to dissociate bottom-up visual saliency and action selection signals, they may not represent entirely separable processes but aspects of the same competition processes implemented throughout the visuomotor system (Cisek \& Kalaska, 2010). Nevertheless, we wanted to see how the direct manipulation of bottom-up visual saliency would affect the choice behavior. Although the (behaviorally irrelevant) visual contrast strongly affected the choice behavior in control conditions, after inactivation, the alleviation of the choice bias by high visual saliency of the contralesional stimulus was considerably weaker than by high reward. This finding is consistent with studies in neglect patients showing that manipulating the visual saliency in the contralesional field by prolonging stimulus duration, increasing size or luminance, improves extinction symptoms, but 
rather modestly (Bays, Singh-Curry, Gorgoraptis, Driver, \& Husain, 2010; Smania, Martini, Prior, \& Marzi, 1996; Di Pellegrino \& De Renzi, 1995; Zihl \& von Cramon, 1979).

These results suggest that the perceptual visibility of contralesional cues was not the main factor guiding the choice behavior after pulvinar inactivation-monkeys still saw them and were willing to select them more frequently if they led to high reward. The much greater effect of reward on behavior after pulvinar inactivation suggests that there may be separate sources of modulation for reward and bottom-up visual saliency and that reward has a greater effect on the decision process. Future studies that parametrically equalize the effects of reward and visual contrast would be required to address this possibility.

However, because stimulus detection and saccade response were not dissociated in the current study, we cannot exclude the possibility that perceptual factors also contributed to the observed deficits. Moreover, in one of the monkeys, the reward modulation did not completely overcome the inactivation-induced spatial bias, suggesting that perceptual extinction of contralesional targets may have occurred in some of the trials.

What could be the neural mechanism for the restoration of contralesional choices when the high reward cue was placed into the contralesional hemifield? There are two main possibilities, which are not mutually exclusive: (1) Modulation of activity in cortical areas that receive direct input from the pulvinar are involved in oculomotor planning and are modulated by reward expectation. Several cortical areas that exchange projections with the dorso-medial pulvinar (e.g., FEF, dlPFC, LIP, and the posterior cingulate) link visual cues with oculomotor behavior and increase their activity with the expected value of potential eye movements (Ding \& Hikosaka, 2006; McCoy, Crowley, Haghighian, Dean, \& Platt, 2003; Leon \& Shadlen, 1999; Platt \& Glimcher, 1999). It is thus possible that high reward expectation elevated activity in those areas within the lesioned hemisphere to a level that triggered a saccade choice into the contralesional field. Assuming that activity in those cortical areas at the side of the lesion was decreased following pulvinar inactivation (Wilke, Kagan, \& Andersen, 2010), the higher reward in the contralesional field, in comparison with the ipsilesional field (represented by the intact hemisphere), may have increased activity in the lesioned hemisphere over the decision threshold. (2) Alternatively, reward could have restored contralesional choices by modulation of reward-sensitive subcortical structures, which do not receive direct projections from the pulvinar. For example, the increase of contralesional choices with higher reward could have been mediated by structures of the oculomotor decision networks that contain spatial maps such as the caudate (Mort et al., 2003), the SC (Lauwereyns, Takikawa, et al., 2002), and the substantia nigra (Sato \& Hikosaka, 2002).

In conclusion, we propose that inactivation of the dorsal pulvinar changes the balance between behavioral desirability of competing spatial locations while leaving the visibility, reward processing, spatial memory, and saccade execution functions largely intact.

\section{Acknowledgments}

We thank K. Pejsa and N. Sammons for animal care and Dr. V. Shcherbatyuk for computer support. This work was supported by Moore Foundation, National Eye Institute, National Science Foundation, Defense Advanced Research Projects Agency, and Boswell Foundation (to R. A.) and the Herman and Lilly Schilling Foundation (to M. W.).

Reprint requests should be sent to Melanie Wilke, University of Goettingen, Robert-Koch-Str. 40, Goettingen, 37075, Germany, or via e-mail: melanie.wilke@med.uni-goettingen.de.

\section{REFERENCES}

Arend, I., Machado, L., Ward, R., McGrath, M., Ro, T., \& Rafal, R. D. (2008). The role of the human pulvinar in visual attention and action: Evidence from temporal-order judgment, saccade decision, and antisaccade tasks. Progress in Brain Research. 171, 475-483.

Arend, I., Rafal, R., \& Ward, R. (2008). Spatial and temporal deficits are regionally dissociable in patients with pulvinar lesions. Brain, 131, 2140-2152.

Asanuma, C., Andersen, R. A., \& Cowan, W. M. (1985). The thalamic relations of the caudal inferior parietal lobule and the lateral prefrontal cortex in monkeys: Divergent cortical projections from cell clusters in the medial pulvinar nucleus. Journal of Comparative Neurology, 241, 357-381.

Baker, J. T., Patel, G. H., Corbetta, M., \& Snyder, L. H. (2006). Distribution of activity across the monkey cerebral cortical surface, thalamus and midbrain during rapid, visually guided saccades. Cerebral Cortex, 16, 447-459.

Baleydier, C., \& Mauguiere, F. (1985). Anatomical evidence for medial pulvinar connections with the posterior cingulate cortex, the retrosplenial area, and the posterior parahippocampal gyrus in monkeys. Journal of Comparative Neurologv, 232, 219-228.

Bays, P. M., Singh-Curry, V., Gorgoraptis, N., Driver, J., \& Husain, M. (2010). Integration of goal- and stimulus-related visual signals revealed by damage to human parietal cortex. Iournal of Neuroscience, 30, 5968-5978.

Bender, D. B., \& Youakim, M. (2001). Effect of attentive fixation in macaque thalamus and cortex. Journal of Neurophysiology, 85, 219-234.

Benevento, L. A., \& Port, J. D. (1995). Single neurons with both form/color differential responses and saccade-related responses in the nonretinotopic pulvinar of the behaving macaque monkey. Visual Neuroscience, 12, 523-544.

Berman, R. A., \& Wurtz, R. H. (2011). Signals conveyed in the pulvinar pathway from superior colliculus to cortical area MT. Iournal of Neuroscience, 31, 373-384.

Blatt, G. J., Andersen, R. A., \& Stoner, G. R. (1990). Visual receptive field organization and cortico-cortical connections of the lateral intraparietal area (area LIP) in the macaque. Lournal of Comparative Neurology, 299, 421-445.

Cappe, C., Morel, A., \& Rouiller, E. M. (2007). Thalamocortical and the dual pattern of corticothalamic projections of the posterior parietal cortex in macaque monkeys. Neuroscience. 146, 1371-1387.

Chafee, M. V., \& Goldman-Rakic, P. S. (1998). Matching patterns of activity in primate prefrontal area $8 \mathrm{a}$ and parietal area 7ip 
neurons during a spatial working memory task. Journal of Neurophysiology, 79, 2919-2940.

Cisek, P., \& Kalaska, J. F. (2010). Neural mechanisms for interacting with a world full of action choices. Annual Review of Neuroscience, 33, 269-298.

Danziger, S., Ward, R., Owen, V., \& Rafal, R. (2001). The effects of unilateral pulvinar damage in humans on reflexive orienting and filtering of irrelevant information. Behavioural Neurology, 13, 95-104.

Di Pellegrino, G., \& De Renzi, E. (1995). An experimental investigation on the nature of extinction. Neuropsychologia. 33, $153-170$

Dias, E. C., \& Segraves, M. A. (1999). Muscimol-induced inactivation of monkey frontal eye field: Effects on visually and memory-guided saccades. Journal of Neurophysiology, 81, 2191-2214.

Ding, L., \& Hikosaka, O. (2006). Comparison of reward modulation in the frontal eye field and caudate of the macaque. Lournal of Neuroscience, 26, 6695-6703.

Dominguez-Borras, J., Saj, A., Armony, J. L., \& Vuilleumier, P. (2012). Emotional processing and its impact on unilateral neglect and extinction. Neuropsychologia. 50, 1054-1071.

Engelmann, J. B., \& Pessoa, L. (2007). Motivation sharpens exogenous spatial attention. Emotion, 7, 668-674.

Fox, E. (2002). Processing emotional facial expressions: The role of anxiety and awareness. Cognitive. Affective $\mathcal{E}$ Behavioral Neuroscience, 2, 52-63.

Funahashi, S., Bruce, C. J., \& Goldman-Rakic, P. S. (1993). Dorsolateral prefrontal lesions and oculomotor delayedresponse performance: Evidence for mnemonic "scotomas." Journal of Neuroscience, 13, 1479-1497.

Gnadt, J. W., \& Andersen, R. A. (1988). Memory related motor planning activity in posterior parietal cortex of macaque. Experimental Brain Research, 70, 216-220.

Grabowska, A., Marchewka, A., Seniow, J., Polanowska, K., Jednorog, K., Krolicki, L., et al. (2011). Emotionally negative stimuli can overcome attentional deficits in patients with visuo-spatial hemineglect. Neuropsvchologia 49, 3327-3337.

Grieve, K. L., Acuna, C., \& Cudeiro, J. (2000). The primate pulvinar nuclei: Vision and action. Trends in Neurosciences. 23, 35-39.

Gutierrez, C., Cola, M. G., Seltzer, B., \& Cusick, C. (2000). Neurochemical and connectional organization of the dorsal pulvinar complex in monkeys. Journal of Comparative Neurologv, 419, 61-86.

Hickey, C., Chelazzi, L., \& Theeuwes, J. (2010). Reward changes salience in human vision via the anterior cingulate. Lournal of Neuroscience, 30, 11096-11103.

Hulme, O. J., Whiteley, L., \& Shipp, S. (2010). Spatially distributed encoding of covert attentional shifts in human thalamus. Journal of Neurophvsiologv, 104, 3644-3656.

Kaas, J. H., \& Lyon, D. C. (2007). Pulvinar contributions to the dorsal and ventral streams of visual processing in primates. Brain Research Reviews, 55, 285-296.

Kagan, I., Iyer, A., Lindner, A., \& Andersen, R. A. (2010). Space representation for eye movements is more contralateral in monkeys than in humans. Proceedings of the National Academv of Sciences. U.S.A., 107, 7933-7938.

Karnath, H. O., Himmelbach, M., \& Rorden, C. (2002). The subcortical anatomy of human spatial neglect: Putamen, caudate nucleus and pulvinar. Brain, 125, 350-360.

Kastner, S., O'Connor, D. H., Fukui, M. M., Fehd, H. M., Herwig, U., \& Pinsk, M. A. (2004). Functional imaging of the human lateral geniculate nucleus and pulvinar. Iournal of Neuropbvsiology, 91, 438-448.

Kinsbourne, M. (1977). Hemi-neglect and hemisphere rivalry. Advances in Neurology, 18, 41-49.
Krebs, R. M., Boehler, C. N., Roberts, K. C., Song, A. W., \& Woldorff, M. G. (2012). The involvement of the dopaminergic midbrain and cortico-striatal-thalamic circuits in the integration of reward prospect and attentional task demands. Cerebral Cortex, 22, 607-615.

Krebs, R. M., Woldorff, M. G., Tempelmann, C., Bodammer, N., Noesselt, T., Boehler, C. N., et al. (2010). High-field fMRI reveals brain activation patterns underlying saccade execution in the human superior colliculus. Plos One, 5, e8691.

Kurniawan, I. T., Guitart-Masip, M., \& Dolan, R. J. (2011). Dopamine and effort-based decision making. Frontiers in Neuroscience, 5, 81.

Lauwereyns, J., Takikawa, Y., Kawagoe, R., Kobayashi, S., Koizumi, M., Coe, B., et al. (2002). Feature-based anticipation of cues that predict reward in monkey caudate nucleus. Neuron, 33, 463-473.

Lauwereyns, J., Watanabe, K., Coe, B., \& Hikosaka, O. (2002). A neural correlate of response bias in monkey caudate nucleus. Nature, 418, 413-417.

Leon, M. I., \& Shadlen, M. N. (1999). Effect of expected reward magnitude on the response of neurons in the dorsolateral prefrontal cortex of the macaque. Neuron, 24, 415-425.

Li, C. S., Mazzoni, P., \& Andersen, R. A. (1999). Effect of reversible inactivation of macaque lateral intraparietal area on visual and memory saccades. Journal of Neurophysiology, 81, 1827-1838.

Liu, Y., Yttri, E. A., \& Snyder, L. H. (2010). Intention and attention: Different functional roles for LIPd and LIPv. Nature Neuroscience, 13, 495-500.

Lucas, N., Diserens, K., Leroy, R., Krattinger, S., \& Vuilleumier, P. (2005). Gambling against neglect: Modulation of spatial attention by implicit reward learning. Journal of Cognitive Neuroscience, Suppl. S, 151.

McCoy, A. N., Crowley, J. C., Haghighian, G., Dean, H. L., \& Platt, M. L. (2003). Saccade reward signals in posterior cingulate cortex. Neuron, 40, 1031-1040.

McCoy, A. N., \& Platt, M. L. (2005). Expectations and outcomes: Decision-making in the primate brain. Journal of Comparative Physiologv A: Neuroethologv. Sensorv. Neural and Behavioral Pbysiology, 191, 201-211.

Mesulam, M. M. (1999). Spatial attention and neglect: Parietal, frontal and cingulate contributions to the mental representation and attentional targeting of salient extrapersonal events. Philosophical Transactions of the Royal Society of London, Series B, Biological Sciences, 354, 1325-1346.

Milstein, D. M., \& Dorris, M. C. (2007). The influence of expected value on saccadic preparation. Journal of Neuroscience, 27, $4810-4818$.

Mort, D. J., Malhotra, P., Mannan, S. K., Rorden, C., Pambakian, A., Kennard, C., et al. (2003). The anatomy of visual neglect. Brain 126, 1986-1997.

Opris, I., \& Bruce, C. J. (2005). Neural circuitry of judgment and decision mechanisms. Brain Research. Brain Research Reviews, 48, 509-526.

Padmala, S., Lim, S. L., \& Pessoa, L. (2010). Pulvinar and affective significance: Responses track moment-to-moment stimulus visibility. Frontiers in Human Neuroscience 4, 64

Padmala, S., \& Pessoa, L. (2010). Interactions between cognition and motivation during response inhibition. Neuropsychologia, 48, 558-565.

Pessoa, L., \& Engelmann, J. B. (2010). Embedding reward signals into perception and cognition. Frontiers in Neuroscience 4, 17

Petersen, S. E., Robinson, D. L., \& Keys, W. (1985). Pulvinar nuclei of the behaving rhesus monkey: Visual responses and their modulation. Journal of Neurophysiology, 54 , 867-886. 
Petersen, S. E., Robinson, D. L., \& Morris, J. D. (1987). Contributions of the pulvinar to visual spatial attention. Neuropsvchologia, 25, 97-105.

Platt, M. L., \& Glimcher, P. W. (1999). Neural correlates of decision variables in parietal cortex. Nature, 400, 233-238.

Pleger, B., Blankenburg, F., Ruff, C. C., Driver, J., \& Dolan, R. J. (2008). Reward facilitates tactile judgments and modulates hemodynamic responses in human primary somatosensory cortex. Journal of Neuroscience, 28, 8161-8168.

Rafal, R. D., \& Posner, M. I. (1987). Deficits in human visual spatial attention following thalamic lesions. Proceedings of the National Academv of Sciences. U.S.A., 84, 7349-7353.

Robinson, D. L., McClurkin, J. W., \& Kertzman, C. (1990). Orbital position and eye movement influences on visual responses in the pulvinar nuclei of the behaving macaque. Experimental Brain Research, 82, 235-246.

Robinson, D. L., McClurkin, J. W., Kertzman, C., \& Petersen, S. E. (1991). Visual responses of pulvinar and collicular neurons during eye movements of awake, trained macaques. Journal of Neurophysiology, 66, 485-496.

Robinson, D. L., \& Petersen, S. E. (1992). The pulvinar and visual salience. Trends in Neurosciences, 15, 127-132.

Saalmann, Y. B., \& Kastner, S. (2011). Cognitive and perceptual functions of the visual thalamus. Neuron, 71, 209-223.

Saleem, K., \& Logothetis, N. K. (2006). A combined MRI and bistology atlas of the rhesus monkey brain. New York: Academic Press.

Sato, M., \& Hikosaka, O. (2002). Role of primate substantia nigra pars reticulata in reward-oriented saccadic eye movement. Journal of Neuroscience, 22, 2363-2373.

Scalaidhe, S. P., Albright, T. D., Rodman, H. R., \& Gross, C. G. (1995). Effects of superior temporal polysensory area lesions on eye movements in the macaque monkey. Journal of Neurophysiology, 73, 1-19.

Schiller, P. H., \& Chou, I. H. (1998). The effects of frontal eye field and dorsomedial frontal cortex lesions on visually guided eye movements. Nature Neuroscience, 1, 248-253.

Selemon, L. D., \& Goldman-Rakic, P. S. (1988). Common cortical and subcortical targets of the dorsolateral prefrontal and posterior parietal cortices in the rhesus monkey: Evidence for a distributed neural network subserving spatially guided behavior. Journal of Neuroscience, 8, 4049-4068.

Sherman, S. M. (2005). Thalamic relays and cortical functioning. Progress in Brain Research, 149, 107-126.

Smania, N., Martini, M. C., Prior, M., \& Marzi, C. A. (1996). Input and response determinants of visual extinction: A case study. Cortex, 32, 567-591.

Soto, D., Funes, M. J., Guzman-Garcia, A., Warbrick, T., Rotshtein, P., \& Humphreys, G. W. (2009). Pleasant music overcomes the loss of awareness in patients with visual neglect. Proceedings of the National Academv of Sciences. U.S.A., 106, 6011-6016.

Stepniewska, I. (2004). The pulvinar complex. In J. H. Kaas \& C. E. Collins (Eds.), The primate visual system (pp. 53-80). London: CRC Press.

Takeda, K., \& Funahashi, S. (2002). Prefrontal task-related activity representing visual cue location or saccade direction in spatial working memory tasks. Journal of Neurophysiology, 87, 567-588

Takikawa, Y., Kawagoe, R., Itoh, H., Nakahara, H., \& Hikosaka, O. (2002). Modulation of saccadic eye movements by predicted reward outcome. Experimental Brain Research, 142, 284-291.

Van der Stigchel, S., Arend, I., van Koningsbruggen, M. G., \& Rafal, R. D. (2010). Oculomotor integration in patients with a pulvinar lesion. Neuropsychologia, 48, 3497-3504.

Vuilleumier, P., Armony, J. L., Clarke, K., Husain, M., Driver, J., \& Dolan, R. J. (2002). Neural response to emotional faces with and without awareness: Event-related fMRI in a parietal patient with visual extinction and spatial neglect. Neurotsvchologia, 40, 2156-2166.

Vuilleumier, P., \& Schwartz, S. (2001). Emotional facial expressions capture attention. Neurology , 56, 153-158.

Wardak, C., Olivier, E., \& Duhamel, J. R. (2002). Saccadic target selection deficits after lateral intraparietal area inactivation in monkeys. Journal of Neuroscience, 22, 9877-9884.

Wilke, M., Kagan, I., \& Andersen, R. A. (2010). Pulvinar inactivation alters cortical responses during spatial decision making (Conference abstract). Society for Neuroscience Meeting, San Diego.

Wilke, M., Kagan, I., \& Andersen, R. A. (2012). Functional imaging reveals rapid reorganization of cortical activity after parietal inactivation in monkeys. Proceedings of the National Academv of Sciences. U.S.A., 109, 8274-8279.

Wilke, M., Mueller, K. M., \& Leopold, D. A. (2009). Neural activity in the visual thalamus reflects perceptual suppression. Proceedings of the National Academv of Sciences. U.S.A., 106, 9465-9470.

Wilke, M., Turchi, J., Smith, K., \& Leopold, D. A. (2010). Pulvinar inactivation disrupts selection of movement plans. Journal of Neuroscience, 30, 8650-8659.

Yeterian, E. H., \& Pandya, D. N. (1989). Thalamic connections of the cortex of the superior temporal sulcus in the rhesus monkey. Iournal of Comparative Neurology 282, 80-97.

Zihl, J., \& von Cramon, D. (1979). The contribution of the "second" visual system to directed visual attention in man. Brain, 102, 835-856. 